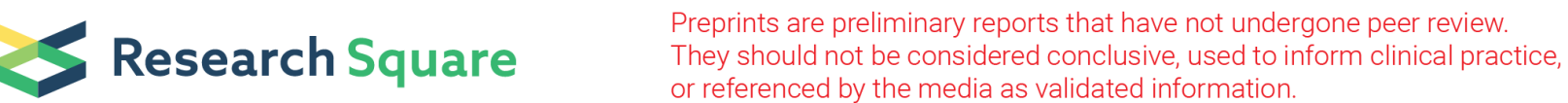

\section{Malignant Gastrointestinal Neuroectodermal Tumor in the Right Atrium: The First Case Report Presenting with an Extra-Gastrointestinal Mass}

\section{Zhiwen Li}

Nanjing Drum Tower Hospital: Nanjing University Medical School Affiliated Nanjing Drum Tower Hospital

Xiaohong Pu

Nanjing Drum Tower Hospital: Nanjing University Medical School Affiliated Nanjing Drum Tower Hospital

Yao Fu

Nanjing Drum Tower Hospital: Nanjing University Medical School Affiliated Nanjing Drum Tower Hospital

Lin Li

Nanjing Drum Tower Hospital: Nanjing University Medical School Affiliated Nanjing Drum Tower Hospital

\section{Yuemei Xu}

Nanjing Drum Tower Hospital: Nanjing University Medical School Affiliated Nanjing Drum Tower Hospital

\section{Xiangshan Fan ( $\sim$ fxs23@163.com )}

Departments of Pathology, the Affiliated Drum Tower Hospital, Nanjing University Medical School, Nanjing 210008, Jiangsu Province, China. https://orcid.org/0000-0002-8728-256X

\section{Case Report}

Keywords: atrium tumor, Malignant gastrointestinal neuroectodermal tumor, EWSR1-ATF1, case report

Posted Date: June 16th, 2021

DOI: https://doi.org/10.21203/rs.3.rs-620292/v1

License: (a) (1) This work is licensed under a Creative Commons Attribution 4.0 International License. Read Full License 


\section{Abstract}

Background: Malignant gastrointestinal neuroectodermal tumor is an extremely rare soft tissue sarcoma which was firstly described in 2003 but until recently it had been designated as a definite new entity. According to the previous literatures, Malignant gastrointestinal neuroectodermal tumor was almost exclusively occurred in gastrointestinal tract.

Case presentation: A 62-year-old male showed a mass in right atrium and the occupying mass was founded on the right ventricular diaphragm and involved the right atrium along the coronary sinus during the operation. Microscopically, the tumor characterized by the solid sheet and pseudopapillary focally architectures, and was composed of small to medium cells with round or oval nuclei, variable amount of eosinophilic or clear, and frequent mitotic figures. Immunohistochemically, the neoplastic cells were positive for S100 and SOX-10 but negative for HMB-45, A103 and CD99. EWSR1-AFTF1 rearrangement was detected by fluorescence in situ hybridization and further confirmed involving fusion of EWSR1 exon 8 with ATF1 exon 4 by whole transcriptome sequence analysis.

Conclusions: This is the first case report of extra-gastrointestinal Malignant gastrointestinal neuroectodermal tumor that occurring in the right atrium $₫$ which remind of the new prospect of the tumor location.

\section{Introduction}

Clear cell sarcoma-like tumor of the gastrointestinal tract (CCSLTGT) or malignant gastrointestinal neuroectodermal tumor (GNET) is a very rare mesenchymal tumor which was first described by Zambrano et al in $2003^{[1]}$. This tumor is much more malignant and prone to local recurrence and metastasis ${ }^{[2]}$. GNET may occurs in any location in the gastrointestinal tract and mainly in small intestine, stomach and colon ${ }^{[3]}$. However, it was almost exclusively occurred in gastroenteric trace, extragastrointestinal GNET has never been reported at present. Here, we reported a case that share the same morphological, immunophenotype and molecular characteristics with GNET originated from the right atrium but not the digestive tract. To our knowledges, this tumor entity has never been reported in this new site before.

\section{Case Presentation}

A 62-year-old male visited our hospital complaining of chest distress and unable to lie down at night for more than one month. He had a history of hypertension but no diabetes, no history of food and drug allergy, major traumatic surgery, hepatitis or tuberculosis, no family history of inherited diseases. On physical examination, there were no special abnormalities in skin.

Computed Tomography (CT) showed filling defect in the right atrial (Fig. 1A) and no abdominal abnormality. Echocardiography revealed a space occupying lesions in right atrial and a moderate pericardial effusion (Fig. 1B). The mass then underwent the surgical excision. The aorta and pulmonary 
artery were in normal arrangement, the diameter of the aorta was about $2.8 \mathrm{~cm}$ and the pulmonary artery about $3.0 \mathrm{~cm}$. The occupying mass was located on the right ventricular diaphragm and involved the right atrium along the coronary sinus. The mass in the right ventricle was $3 \mathrm{~cm} * 3 \mathrm{~cm} * 2 \mathrm{~cm}$ in size and located on the diaphragmatic surface of the right ventricle and infiltrated into the ventricular myocardium with unclear boundary. And the mass in the right atrium was about $4.7 \mathrm{~cm} * 4.3 \mathrm{~cm}$ in size. Full body CT scan was performed and no other mass was found, especially at gastrointestinal tract.

Macroscopically, the cutting surface of the resected tumor showed grey-red and the texture is soft. Microscopically, the tumor was in the pattern of flake distribution (Fig. 2A) or nodular distribution with fiber segmentation (Fig. 2B), and focally exhibited loose reticular structure with pseudopapillomatous formation (Fig. 2C). Iron staining proved that the accumulation of hermosiderine smeary distributed in the tumor (Fig. 2D). The tumor were composed of small to medium cells with round or oval nuclei, fine chromatin, small nucleolus, variable amount of eosinophilic or clear cytoplasm, active mitotic figures and indistinct cell borders (Fig. 2A). Scattered osteoclast-like multinucleated giant cells were not identified.

Immunohistochemically, the neoplastic cells were strongly and diffusely positive for S100 (Fig. 3A) and SOX-10 (Fig. 3B) protein. They were also moderate positive for Syn (Fig. 3C). The proliferation index of tumor cells by the Ki-67 immunostaining was $40 \%$ (Fig. 3D). However, the tumor cells were negative against for HMB45 (Fig. 3E), A103(Fig. 3F) or CD99 (Fig. 3G). The loss expression of INI1 was not identified (Fig. 3H).

Fluorescein in situ hybridization (FISH) analysis for EWSR1 break-apart probe (Fig. 4A) and EWSR1-ATF1 dichromatic probe (Fig. 4B) on paraffin-embedded tumor showed evidence of an EWSR1/22q12 rearrangement. Besides, the whole transcriptome sequencing analysis confirmed that the fusion was involved of exon 8 of EWSR1 and exon 4 of ATF1 (Fig. 4C).

Combined with the clinical presentation, histological, immunophenotypical and molecular genetic characteristics, the diagnose of GENT was made finally.

The patient refused to receive chemotherapy or radiotherapy after the surgery. One month later, the patient was found to have a local recurrence mass, and then died of the disease 20 months later.

\section{Discussion}

Malignant tumors of the atrium are uncommon and the most frequent primary cardiac sarcoma was intimal sarcoma and angiosarcomas ${ }^{[4]}$. However, tumors like the present case which shows the histological characterized by the solid sheet and pseudopapillary focally architectures, immunohistochemically positive for S100 and SOX-10 but negative for HMB-45, A103 and CD99, and appears EWSR1 gene rearrangement by FISH have never been reported in atrium. Based on the histological, immunophenotypical and molecular genetic characteristics, we considered the diagnostic of GNET which was exclusively occurred in the gastrointestinal tract in the past reported references. To test of our conjecture, FISH examination with the EWSR1-ATF1 dichromatic probe and whole transcriptome 
sequencing in paraffin embedded tissue of this tumor were performed respectively, and the results showed that the tumor cells were found to harbor the EWSR1-ATF1 fusion gene, and then to be confirmed by the whole transcriptome sequencing analysis with involving of exon 8 of EWSR1 and exon 4 of ATF1. These findings confirmed our hypothesis and supported the diagnosis of GNET although the extragastrointestinal GNET had never been reported. In the present case, the scattered osteoclast-like multinucleated giant cells, which were only reported in up to $50 \%$ of cases ${ }^{[1,5-8]}$, were not found yet. Besides, no other suspicious mass was found by the full body CT scan examination, including at the abdomen and gastrointestinal tract, suggesting that this tumor was primary but not the secondary tumor at the atrium.

The major differential diagnostic consideration of the present case was clear cell sarcoma (CCS) of soft tissue, melanoma, malignant peripheral nerve sheath tumor (MPNST), Ewing sarcoma / primary neuroectodermal tumor (ES/PNET) and intimal sarcoma (IS). CCS and GNET may share some same histological and immunophenotypically features, and the two tumors can both harbor EWSR1-ATF1 fusion gene. However, CCS often shows melanocyte differentiation and is lack of neuroectodermal differentiation. Because of the accumulation of hemosiderin rather than melanin, and the absence of HMB45 and A103 immunostaining activity but positive for SOX10 in neoplasm cells, the diagnosis of CCS could be ruled out. Because of the expression of S100 protein reactivity and pigment like particle deposition, melanoma also need to be considered. But there were no special abnormalities in skin of this patient and both the melanocytic markers of HMB45 and A103 were negative, these features were useful to rule out melanoma. MPNST should be entered in the differential diagnosis because of similar histological features (spindle and/or epithelioid cells) and S100 protein immunoreactivity. However, MPNST usually doesn't show strong and diffuse immunoreactivity for S100 protein, and genetic study for EWSR1 may help to separate GNET from MPNST [9]. ES/PNET present the similar morphology and protein expression of Syn and CD56 in GNET, however, ES/PNET often shows expression of CD 99 protein and genetic alterations of EWSR1-Fli1 gene fusion ${ }^{[10]}$. Finally, because of the location at the right ventricle of the present tumor, IS which is the most frequent primary cardiac sarcoma, must be excluded. IS often demonstrates MDM2 amplification and negative expression of S100 or SOX10 ${ }^{[4]}$.

It has been widely accepted that GNET is a unique clinicopathological entity with an origin from a gastrointestinal tract. According to Chang's study ${ }^{[9]}$, GNET occurs typically arising in the small intestine with a predilection for the ileum (57.9\%), followed by the stomach (15.8\%), colon (10.5\%), ileocecal junction (5.3\%), lower esophagus (5.3\%), and anal canal (5.3\%). In addition, there were also five cases of GNET have been reported including parapharyngeal and tongue location ${ }^{[1,10-12]}$. Although GNET may occurs in any location in the gastrointestinal tract, it was almost exclusively occurred in gastroenteric trace, extra-gastrointestinal GNET has never been reported at present. In this study, we reported a case that share almost all the characteristics with GNET, which not occurred in the digestive tract but in the right atrium. As far as we know, this is the first reported case of extra-gastrointestinal GNET. 
GNET is a highly malignant tumor and prone to local recurrence and metastasis ${ }^{[3,9]}$. The treatment of GNET was dominated by surgical resection, and a small number of patients showed partial response to apatinib and anlotinib ${ }^{[9]}$. The patient in our case did not receive chemotherapy or radiotherapy, and presented with local recurrence for only one months after diagnosis and then died of the disease 20 months later, suggested that this tumor entity had a very poor prognosis in the extra-gastrointestinal. However, owing to the low incidence, the treatment of GNET still at the elementary step and no evidencebased guidelines, and need further research and exploration.

\section{Conclusions}

In summary, we reported the first case of extra-gastrointestinal GNET arising in the right atrium of a 62year-old male. This novel case adds to the new prospect of the tumor location of GNET.

\section{Abbreviations}

CCSLTGT

Clear cell sarcoma-like tumor of the gastrointestinal tract;

GNET

Malignant gastrointestinal neuroectodermal tumor;

CT

Computed Tomography;

$\mathrm{FISH}$

Fluorescein in situ hybridization;

CCS

Clear cell sarcoma

MPNST

Malignant peripheral nerve sheath tumor;

ES/PNET

Ewing sarcoma / primary neuroectodermal tumor;

IS

Intimal sarcoma.

\section{Declarations}

\section{Acknowledgments}

Not applicable.

\section{Author Contributions}

$\mathrm{ZL}$ and $\mathrm{XP}$ analyzed and interpreted the patient data and was a major contributor in writing the manuscript. YF and LL collected all the clinical and pathological data. YX collected and edited the image. 
FXS revised the final manuscript. All authors contributed to the article and approved the submitted version.

\section{Funding}

None.

\section{Availability of data and materials}

The authors confirm that the data supporting the findings of this study are available within the article.

\section{Ethics approval and consent to participate}

Patient provided informed consent; the present report was approved by the Ethics Committee of the Affiliated Drum Tower Hospital, Nanjing University Medical School.

\section{Consent for publication}

All authors consent to the publication of the manuscript in Diagnostic Pathology.

\section{Competing interests}

The authors declare that they have no competing interests

\section{References}

1. Zambrano E, Reyes-Mugica M, Franchi A. An osteoclast-rich tumor of the gastrointestinal tract with features resembling clear cell sarcoma of soft parts: reports of 6 cases of a GIST simulator. Int $J$ Surg Pathol 2003; 11:75-81.

2. Insabato L, Guadagno $E$, Natella $V$, et al. An unusual association of malignant gastrointestinal neuroectodermal tumor (clear cell sarcoma-like) and Ewing sarcoma. Pathol Res Pract 2015; 211:688-692.

3. Stockman DL, Miettinen M, Suster S, et al. Malignant gastrointestinal neuroectodermal tumor: clinicopathologic, immunohistochemical, ultrastructural, and molecular analysis of 16 cases with a reappraisal of clear cell sarcoma-like tumors of the gastrointestinal tract. Am J Surg Pathol 2012; 36:857-868.

4. Neuville A, Collin F, Bruneval $P$, et al. Intimal sarcoma is the most frequent primary cardiac sarcoma: clinicopathologic and molecular retrospective analysis of 100 primary cardiac sarcomas. Am J Surg Pathol 2014; 38:461-469.

5. Wang J. Clear cell sarcoma-like tumor of the gastrointestinal tract: an evolving entity. Arch Pathol Lab Med 2015; 139:407-412.

6. Antonescu CR, Nafa K, Segal NH, et al.EWS-CREB1: a recurrent variant fusion in clear cell sarcoma-association with gastrointestinal location and absence of melanocytic differentiation. Clin Cancer 
Res 2006; 12:5356-5362.

7. Pauwels P, Debiec-Rychter M, Sciot R, et al. Clear cell sarcoma of the stomach. Histopathology 2002; 41:526-530.

8. Sbaraglia M, Righi A, Gambarotti M. Ewing sarcoma and Ewing-like tumors. Virchows Arch 2020; 476:109-119.

9. Chang B, Yu L, Guo WW, et al. Malignant Gastrointestinal Neuroectodermal Tumor: Clinicopathologic, Immunohistochemical, and Molecular Analysis of 19 Cases. Am J Surg Pathol 2020; 44:456-466.

10. Yegen G, Gulluoglu M, Mete 0 , et al: Clear cell sarcoma-like tumor of the gastrointestinal tract: a case report and review of the literature. Int J Surg Pathol 2015, 23(1):61-67.

11. Kim SB, Lee SH, Gu MJ: Esophageal subepithelial lesion diagnosed as malignant gastrointestinal neuroectodermal tumor. World J Gastroentero/ 2015, 21(18):5739-5743.

12. Gahanbani Ardakani A, Boyle DJ, Elton C: Gastrointestinal clear cell sarcoma-like tumour of the ascending colon. Ann R Coll Surg Engl 2016, 98(3):e37-39.

\section{Figures}
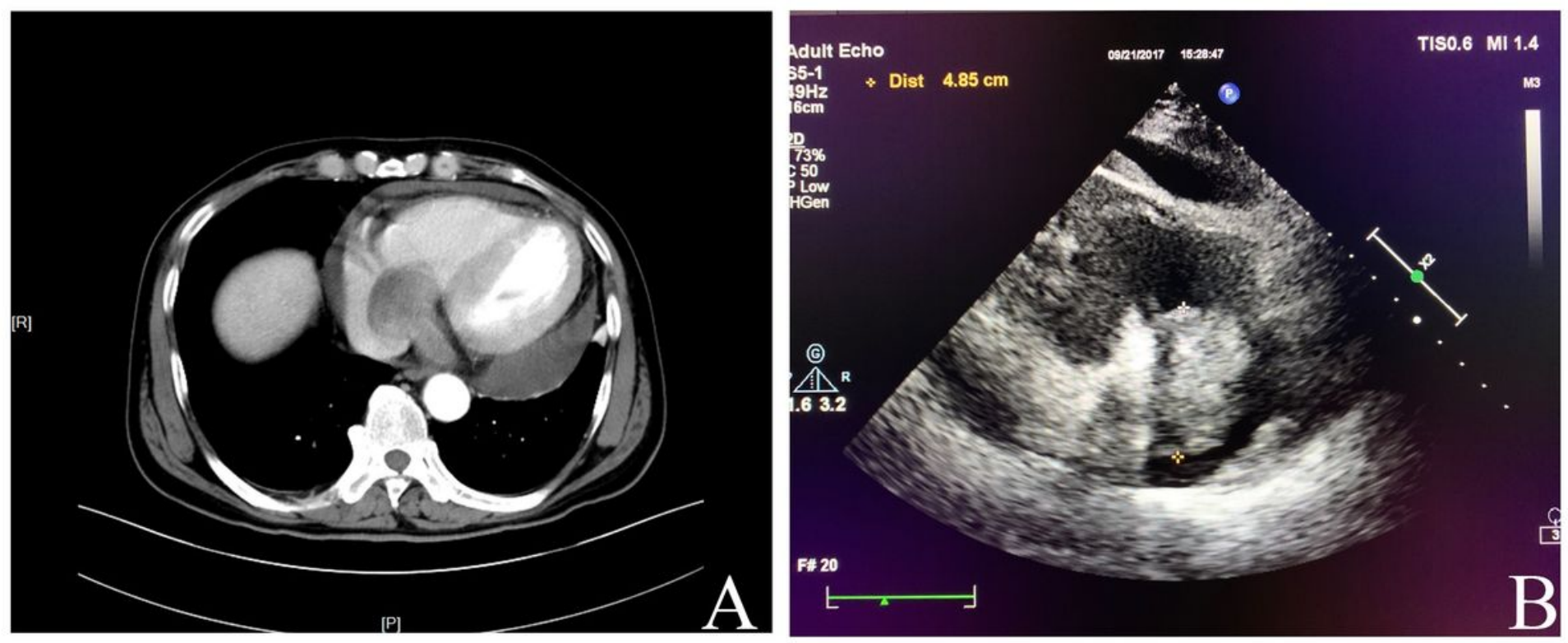

\section{Figure 1}

(A)Computed tomography showed filling defect in the Right atrial. (B)Echocardiography revealed a space occupying lesions in right atrial and a moderate pericardial effusion. 

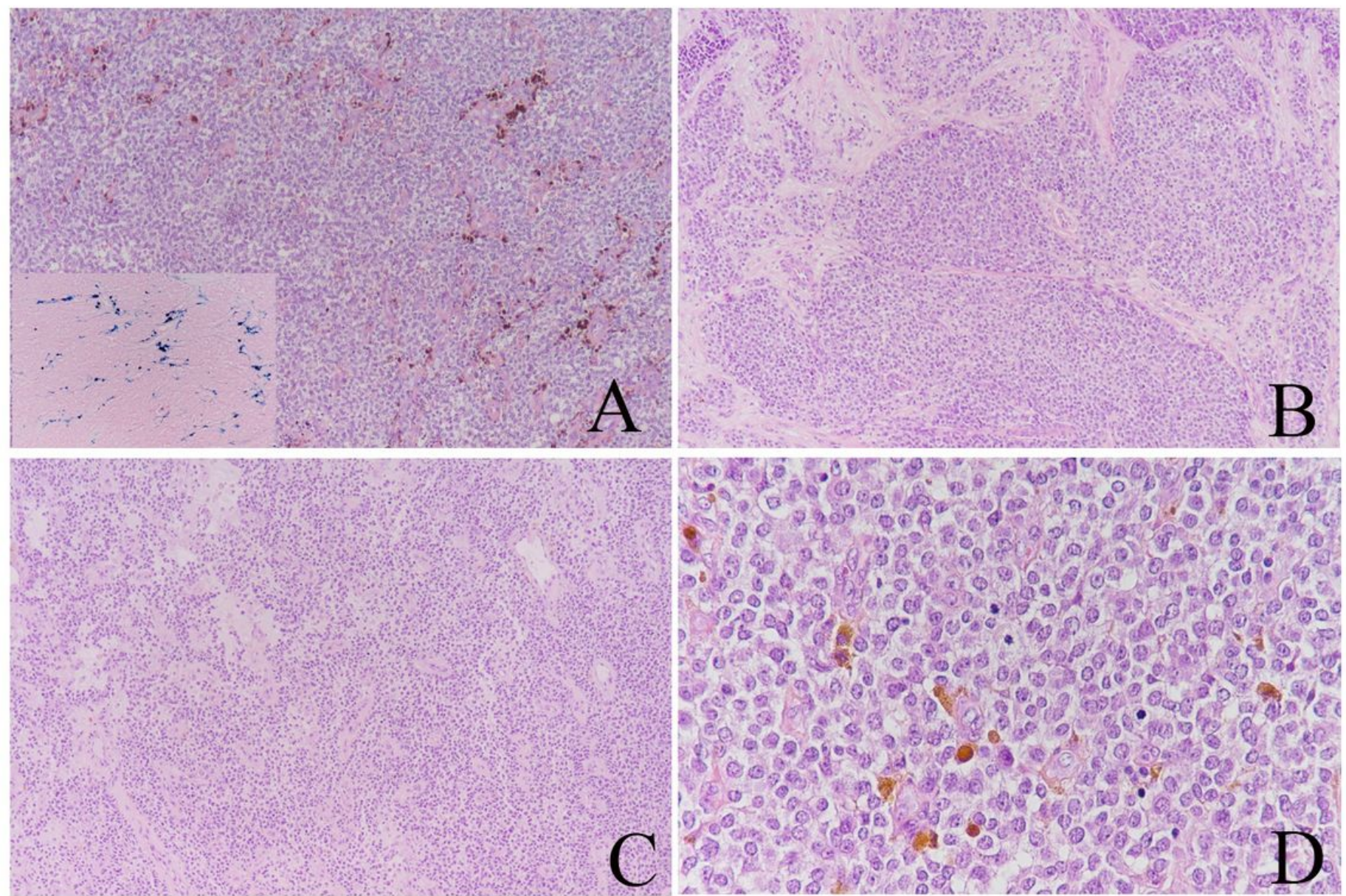

Figure 2

HE staining showed the morphology of GNET. (A) The tumor was in the pattern of diffuse flake with pigment like particles scatter distributing, iron staining (lower-left)showed that these pigment were hemosiderin.(100x); (B)The tumor showed nodular distribution with fiber segmentation(100x);(C) In certain part the neoplasm cells exhibited porous network and pseudopapillomatous structure (100x); (D) In high power, the tumor was composed of small to medium cells with round or oval nuclei, fine chromatin, small nucleolus, variable amount of eosinophilic or clear cytoplasm, and frequent mitotic figures(400x). 

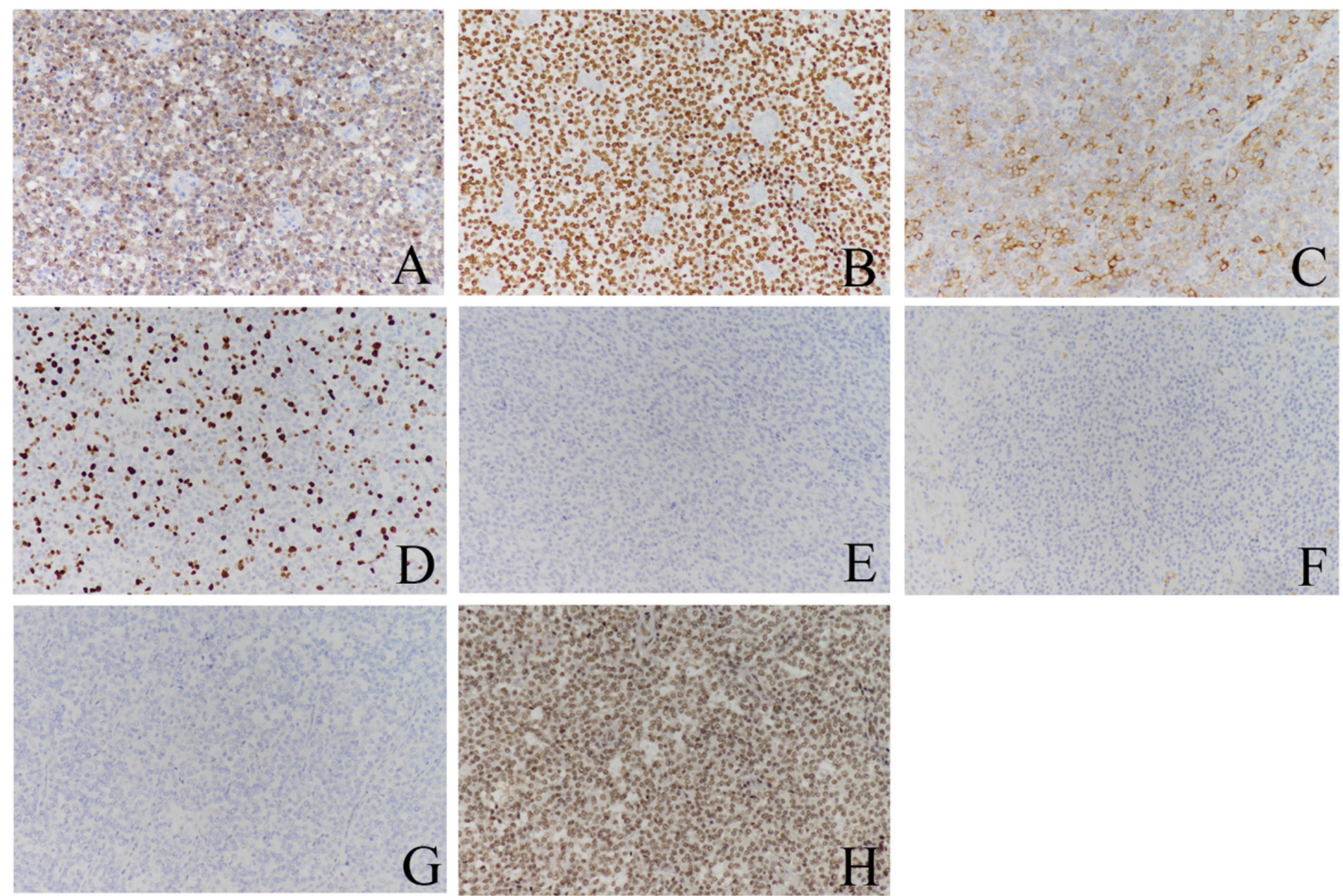

Figure 3

Immunohistochemistry staining of GNET and gastric tumor. (A) The GNET cells were strongly and diffusely positive for S100 and (B) SOX10 staining (100x); (C) moderate positive for Syn; (D) the proliferating cell nuclear antigen Ki-67 was 40\%; (E) HMB45, (F) A103 and (G) CD99 were all negative in the neoplastic cells; $(H)$ The loss expression of INI1 was not identified. 

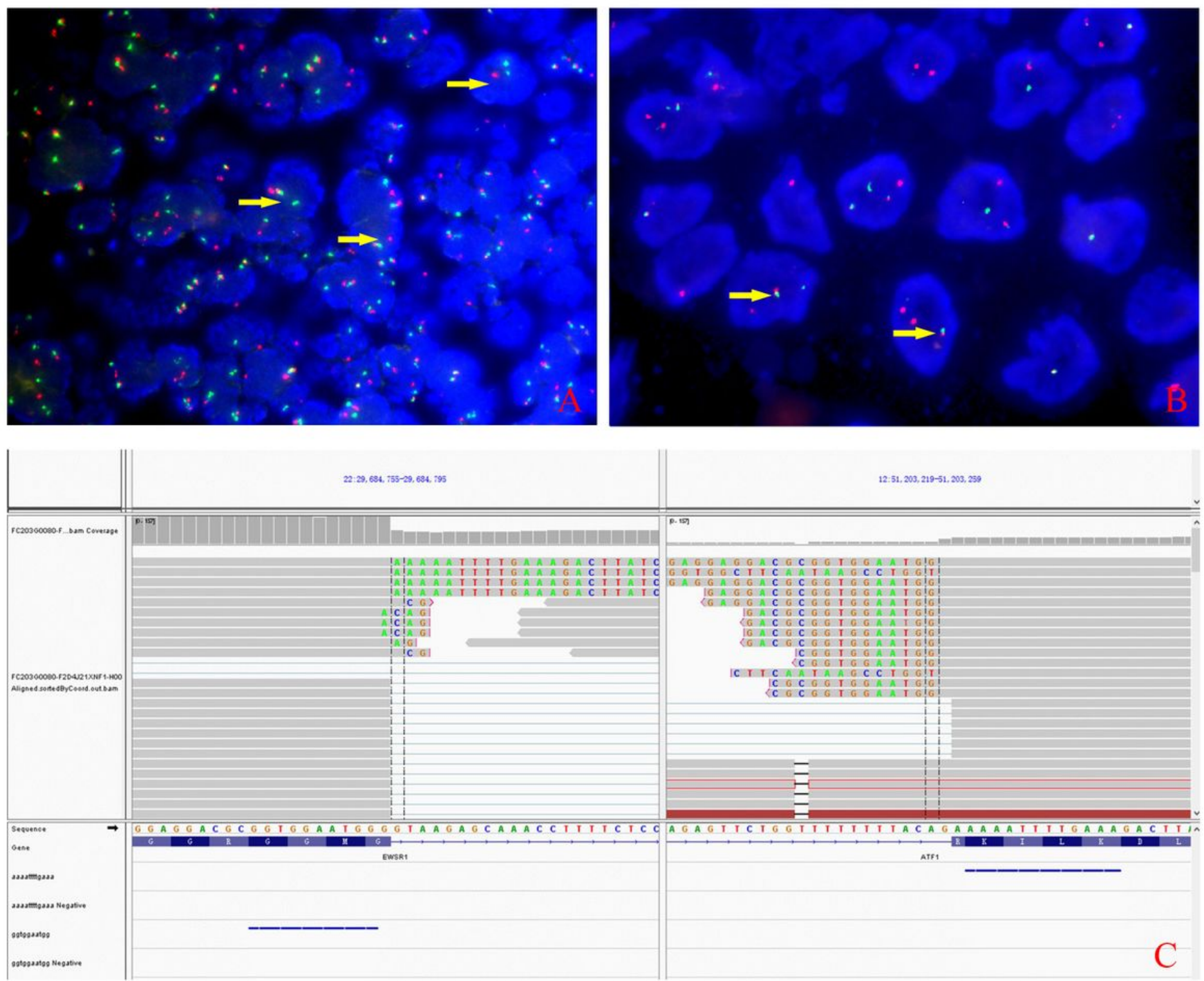

\section{Figure 4}

Molecular genetic characteristics of the GNET. (A) Rearrangement of EWSR1(arrows) was found in about $50 \%$ tumor cells by FISH; (B) EWSR1-AFTF1 dichromatic probe further proved the tumor carrying EWSR1

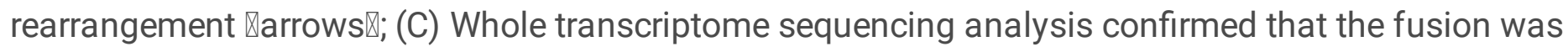
involved of EWSR1 exon8 with ATF1 exon4. 